\title{
Microscopic scale modelling of fluids and solutes confined in unsaturated clays
}

\author{
VIRGINIE MARRY ${ }^{1}$, SÉBASTIEN LE CROM ${ }^{1,2}$, \\ CHRISTOPHE TOURNASSAT ${ }^{3,4,5}$ AND JEAN-CHARLES \\ ROBINET $^{2}$
}

${ }^{1}$ Sorbonne Université, UPMC Univ Paris 06, UMR 8234

${ }^{2}$ ANDRA

${ }^{3}$ BRGM

${ }^{4}$ University of Orléans

${ }^{5}$ Lawrence Berkeley National Laboratory

Presenting Author: sebastien.lecrom@cea.fr

Because of their many applications, clays have been widely studied both experimentally and theoretically. In particular, their low permeability make them key materials in deep geological disposal facilities for radioactive waste management such as the Cigéo project in France [1]. Under repository conditions clay materials are also expected to be partially saturated with potential effects on solutes mobility. Because of the influence of shortrange interactions in pores of few nanometers, Molecular Dynamics (MD) constitute a powerful tool to study the impact of charged surfaces on the species diffusion mechanisms inside the pores. However, a decisive step in MD simulations is the choice of the force field, which models the interactions between atoms. We already showed that explicitely taking into account the atomic polarizability had a significant influence on the properties of aqueous species at the clay interface under saturated conditions [2].

In this study, montmorillonite pores under various unsaturated conditions are simulated using PIM the polarizable force field developed by PHENIX group [3]. We show that the species mobility is greatly reduced with increasing unsaturation of the pore, especially for ions. At large unsaturation an overcompensation of the clay charge is observed by cation condensation at a short distance from the surface. Introducing the polarizability leads to a stronger repulsion of the cations from the surface compared to the non polarizable force field ClayFF [4]. The cations are thus less localized next to surface sites, which greatly changes the wetting properties of the surface. Indeed, while the surface remains fully hydrated with the PIM polarizable force field, droplets disconnected from each other are formed on the clay surface with the ClayFF force field. This may have a strong influence on the mobility of aqueous species for the greatest unsaturations.

[1] ANDRA (2005) Report CRPADS040022_B, Tome 2

[2] Le Crom S.; Tournassat C.; Robinet J.-C.; Marry V. (2020), J. Phys Chem C 124, 11, 6221-6232

[3] Tesson S.; Salanne M.; Rotenberg B.; Tazi S.; Marry V. (2016), J. Phys. Chem. C 120, 7, 3749-3758

[4] Cygan T.; Liang J.; Kalinichev A. (2004), J. Phys. Chem. $B, 108,1255-1266$ 